Supporting Information

\title{
Exploring the interaction mechanism of desmethyl- broflanilide in insect GABA receptors and screening potential antagonists by in silico simulations
}

\author{
Ya Gao $^{\mathrm{a}, \dagger}$, Yichi Zhang ${ }^{\mathrm{b}, \dagger}$, Fengshou Wu ${ }^{\mathrm{a}}$, Jianfeng Pei ${ }^{\mathrm{c}}$, Xiaogang Luo ${ }^{\mathrm{a}, \mathrm{d}}$, \\ Xiulian $\mathrm{Ju}^{\text {a }}$, Chunqing Zhao ${ }^{\mathrm{b}, *}$, Genyan Liu ${ }^{\mathrm{a}, *}$
}

${ }^{\text {a } H u b e i ~ K e y ~ L a b o r a t o r y ~ o f ~ N o v e l ~ R e a c t o r ~ a n d ~ G r e e n ~ C h e m i c a l ~ T e c h n o l o g y, ~ S c h o o l ~ o f ~}$ Chemical Engineering and Pharmacy, Wuhan Institute of Technology, Wuhan 430205, P. R. China

${ }^{\mathrm{b}}$ Education Ministry Key Laboratory of Integrated Management of Crop Diseases and Pests, College of Plant Protection, Nanjing Agricultural University, Nanjing, 210095, P. R. China

${ }^{\mathrm{c}}$ Center for Quantitative Biology, Academy for Advanced Interdisciplinary Studies, Peking University, Beijing 100871, P. R. China

${ }^{\mathrm{d}}$ School of Materials Science and Engineering, Zhengzhou University, Zhengzhou 450001, P. R. China

†Y.G. and Y.Z. contributed equally to this study.

*Corresponding authors: Genyan Liu, Chunqing Zhao

E-mail addresses: liugenyan@yahoo.com (G. Liu),zcq@njau.edu.cn (C. Zhao) 


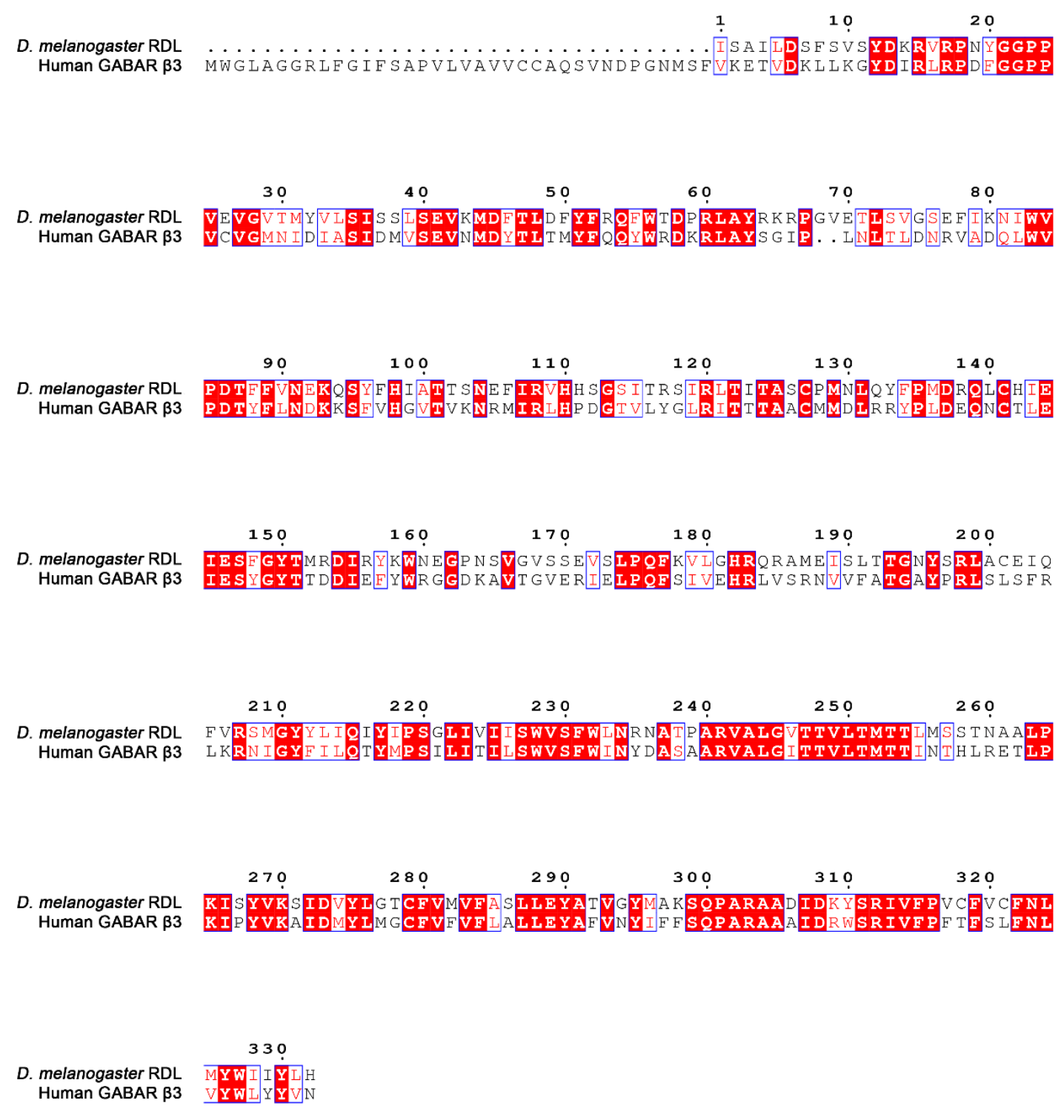

Figure S1. Sequence alignment of the human $\mathrm{GABA}_{\mathrm{A}} \beta 3$ subunit and the $D$. melanogaster RDL subunit. 


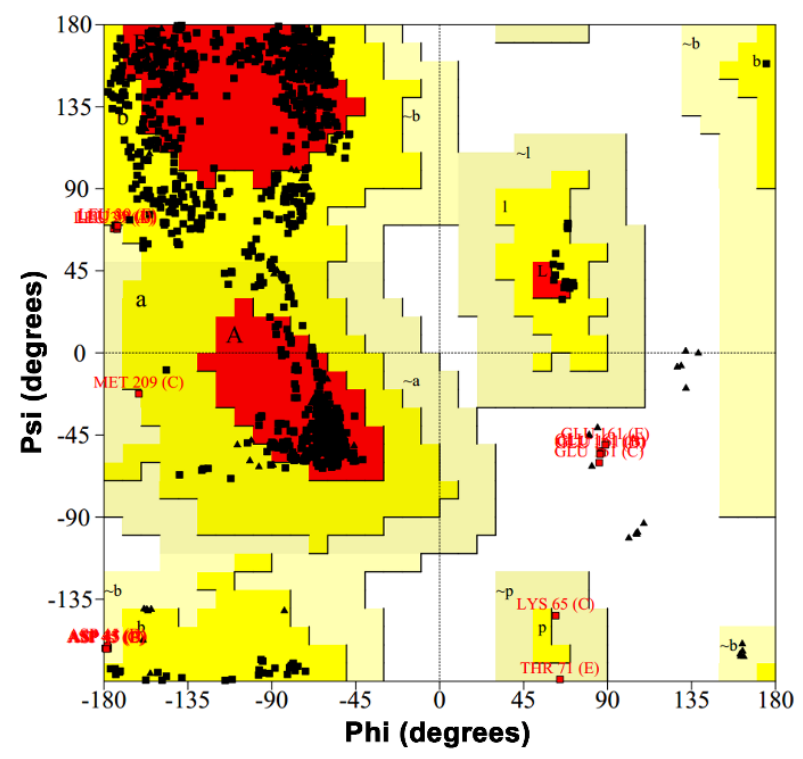

Figure S2. Ramachandran plots of the D. melanogaster RDLR model obtained directly by homology modeling. 


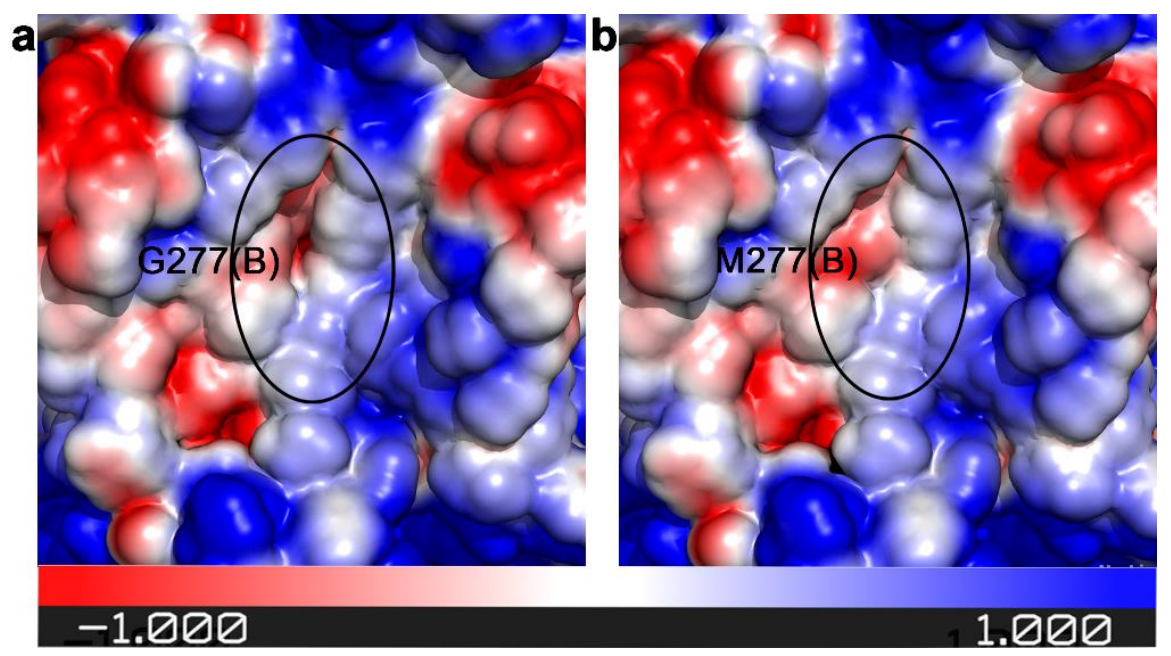

Figure S3. Surface electrostatic potential distributions of the docking pockets of the $D$. melanogaster WT (a) and G277M (b) RDLR models. The positive potential is colored in blue; the negative potential is colored in red; the mutation site is circled by black oval. 
Table S1. Docking scores of top 10 DMBF conformations in the D. melanogaster WT RDLR model.

\begin{tabular}{lcc}
\hline No. & Total Score & CScore \\
\hline 01 & 5.3715 & 5 \\
02 & 4.8119 & 4 \\
03 & 4.6213 & 3 \\
04 & 4.1879 & 3 \\
05 & 4.1340 & 1 \\
06 & 4.0774 & 1 \\
07 & 3.2226 & 2 \\
08 & 3.1483 & 0 \\
09 & 3.0682 & 0 \\
10 & 2.9735 & 2 \\
\hline
\end{tabular}


Table S2. Statistical results of the constructed twenty pharmacophore models.

\begin{tabular}{|c|c|c|c|c|c|c|c|c|}
\hline Name & SPECIFICITY & N_HITS & FEATS & PARETO & ENERGY & STERICS & HBOND & MOL_QRY \\
\hline MODEL_1 & 4.547 & 11 & 8 & 0 & 18.79 & 4548.00 & 347.70 & 305.53 \\
\hline MODEL_2 & 5.488 & 6 & 9 & 0 & 19.82 & 4449.20 & 346.10 & 333.62 \\
\hline MODEL_3 & 4.547 & 11 & 8 & 0 & 18.50 & 4943.60 & 319.10 & 244.66 \\
\hline MODEL_4 & 5.830 & 9 & 9 & 0 & 16.86 & 4112.20 & 342.40 & 242.25 \\
\hline MODEL_5 & 5.835 & 10 & 8 & 0 & 17.69 & 3868.10 & 348.20 & 305.88 \\
\hline MODEL_6 & 4.545 & 9 & 8 & 0 & 15.98 & 3796.80 & 328.50 & 284.33 \\
\hline MODEL_7 & 5.577 & 9 & 9 & 0 & 17.84 & 4103.20 & 340.70 & 293.31 \\
\hline MODEL_8 & 5.575 & 10 & 9 & 0 & 23.40 & 4622.50 & 341.20 & 250.47 \\
\hline MODEL_9 & 5.801 & 8 & 8 & 0 & 17.94 & 4607.80 & 319.20 & 273.66 \\
\hline MODEL_10 & 4.509 & 10 & 8 & 0 & 24.39 & 4657.90 & 333.60 & 284.15 \\
\hline MODEL_11 & 5.490 & 9 & 9 & 0 & 18.85 & 3984.90 & 356.80 & 307.63 \\
\hline MODEL_12 & 4.514 & 10 & 8 & 0 & 17.96 & 4116.40 & 350.80 & 238.53 \\
\hline MODEL_13 & 3.585 & 11 & 8 & 0 & 18.41 & 4535.90 & 342.50 & 232.30 \\
\hline MODEL_14 & 5.488 & 7 & 9 & 0 & 20.07 & 4348.10 & 358.70 & 242.49 \\
\hline MODEL_15 & 5.485 & 6 & 9 & 0 & 16.75 & 4709.20 & 304.00 & 234.19 \\
\hline MODEL_16 & 5.583 & 11 & 9 & $\mathbf{0}$ & 17.52 & 4428.20 & 330.80 & 225.88 \\
\hline MODEL_17 & 4.266 & 10 & 9 & 0 & 20.78 & 4689.80 & 333.30 & 238.08 \\
\hline MODEL_18 & 5.490 & 7 & 9 & 0 & 17.07 & 4271.10 & 328.70 & 238.53 \\
\hline MODEL_19 & 4.512 & 9 & 8 & 0 & 16.26 & 4314.60 & 309.50 & 268.81 \\
\hline MODEL_20 & 4.545 & 11 & 8 & 0 & 18.27 & 4375.50 & 345.50 & 228.21 \\
\hline
\end{tabular}




\section{Purchase information and characterization data of compounds VS1 and VS2}

Compounds VS1 and VS2 were purchased from Crea-Chim UAB (Vilnius, Lithuania) and WuXi AppTec Co., Ltd. (Tianjin, China), respectively.

\section{2-(4-Methoxybenzamido)-N-(3-methyl-1-(2-(4-methylbenzoyl)hydrazineyl)-}

1-oxobutan-2-yl)benzamide (VS1): white solid; ${ }^{1} \mathrm{H}$ NMR $\left(400 \mathrm{MHz}, \mathrm{DMSO}-d_{6}\right) \delta$

$12.17(\mathrm{~s}, 1 \mathrm{H}, \mathrm{CONH}), 10.32(\mathrm{~s}, 1 \mathrm{H}, \mathrm{N}-\mathrm{NH}), 10.21(\mathrm{~s}, 1 \mathrm{H}, \mathrm{N}-\mathrm{NH}), 8.88(\mathrm{~d}, J=8.00 \mathrm{~Hz}$, 1H, C-NH), 8.52 (d, $J=8.00 \mathrm{~Hz}, 1 \mathrm{H}, \mathrm{ArH}), 7.94(\mathrm{~m}, 3 \mathrm{H}, \mathrm{ArH}), 7.79(\mathrm{~d}, J=8.00 \mathrm{~Hz}$, 2H, ArH), $7.58(\mathrm{t}, J=8.00,8.00 \mathrm{~Hz}, 1 \mathrm{H}, \mathrm{ArH}), 7.29(\mathrm{~d}, J=8.00 \mathrm{~Hz}, 2 \mathrm{H}, \mathrm{ArH}), 7.21(\mathrm{t}$, $J=8.00,8.00 \mathrm{~Hz}, 1 \mathrm{H}, \mathrm{ArH}), 7.13(\mathrm{~d}, J=8.0 \mathrm{~Hz}, 2 \mathrm{H}, \mathrm{ArH}), 4.48(\mathrm{t}, J=8.00,8.00 \mathrm{~Hz}$, $1 \mathrm{H}, \mathrm{N}-\mathrm{CH}), 3.83\left(\mathrm{~s}, 3 \mathrm{H}, \mathrm{OCH}_{3}\right), 2.37\left(\mathrm{~s}, 3 \mathrm{H}, \mathrm{ArCH}_{3}\right), 2.23(\mathrm{~m}, 1 \mathrm{H}, \mathrm{CH}), 1.10(\mathrm{~d}, J=$ $\left.8.00 \mathrm{~Hz}, 3 \mathrm{H}, \mathrm{CH}_{3}\right), 1.01\left(\mathrm{~d}, J=4.00 \mathrm{~Hz}, 3 \mathrm{H}, \mathrm{CH}_{3}\right) .{ }^{13} \mathrm{C} \mathrm{NMR}\left(\mathrm{DMSO}-d_{6}\right) \delta 170.55$, $169.24,165.69,164.45,163.72,142.26,139.86,132.82,130.08,129.43,129.41$, $127.92,127.09,123.03,120.68,114.68,58.21,55.96,30.46,21.47,19.64$; MS (ESI+): $m / z 503.2[\mathrm{M}+\mathrm{H}]^{+}, 525.2[\mathrm{M}+\mathrm{Na}]^{+}$.

5-(2-Bromobenzamido)-N-(2-methoxyethyl)-2-(4-methylpiperidin-1-yl)benzamide (VS2): white solid; ${ }^{1} \mathrm{H}$ NMR (400 MHz, $\left.\mathrm{CDCl}_{3}\right) \delta 10.58(\mathrm{~s}, 1 \mathrm{H}, \mathrm{NH}), 8.48(\mathrm{~s}$, $1 \mathrm{H}, \mathrm{NH}), 8.36(\mathrm{t}, J=8.80,2.00 \mathrm{~Hz}, 1 \mathrm{H}, \mathrm{ArH}), 8.08(\mathrm{~s}, 1 \mathrm{H}, \mathrm{ArH}), 7.63(\mathrm{~d}, J=8.00 \mathrm{~Hz}$, 2H, ArH), $7.32(\mathrm{~m}, 3 \mathrm{H}, \mathrm{ArH}), 3.49$ (t, $\left.J=4.80,5.20 \mathrm{~Hz}, 2 \mathrm{H}, \mathrm{CH}_{2}\right), 3.42$ (q, $J=4.80$, 5.20, $\left.5.60 \mathrm{~Hz}, 2 \mathrm{H}, \mathrm{NCH}_{2}\right), 3.39\left(\mathrm{~s}, 3 \mathrm{H}, \mathrm{OCH}_{3}\right), 3.06\left(\mathrm{~d}, J=11.60 \mathrm{~Hz}, 2 \mathrm{H}, \mathrm{CH}_{2}\right), 2.75$ (t, $\left.J=11.60,11.20 \mathrm{~Hz}, 2 \mathrm{H}, \mathrm{CH}_{2}\right), 1.78\left(\mathrm{~d}, J=12.00 \mathrm{~Hz}, 2 \mathrm{H}, \mathrm{CH}_{2}\right), 1.46(\mathrm{~m}, 3 \mathrm{H}, \mathrm{CH}$, $\left.\mathrm{CH}_{2}\right), 1.03\left(\mathrm{~d}, J=6.40 \mathrm{~Hz}, 3 \mathrm{H}, \mathrm{CH}_{3}\right) ;{ }^{13} \mathrm{C} \mathrm{NMR}\left(\mathrm{CDCl}_{3}\right) \delta 166.03,165.98,148.84$, $138.34,134.95,133.37,131.32,129.63,127.96,127.55,124.02,122.82,121.95,119.67$ 71.21, 58.64, 54.36, 39.09, 34.49, 30.32, 21.85; MS (ESI+): $m / z$ 476.1 [M+3] ${ }^{+}$. 